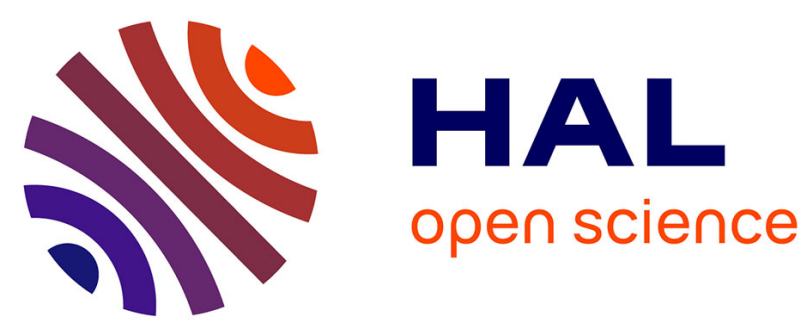

\title{
Probing the cooperative dynamics varying the side-chain length of poly(alkyl acrylate)s: ESR experiments
}

Laura Andreozzi, Ciro Autiero, Massimo Faetti, Marco Giordano, Fabio Zulli

\section{To cite this version:}

Laura Andreozzi, Ciro Autiero, Massimo Faetti, Marco Giordano, Fabio Zulli. Probing the cooperative dynamics varying the side-chain length of poly(alkyl acrylate)s: ESR experiments. Philosophical Magazine, 2007, 87 (3-5), pp.799-810. 10.1080/14786430601003858 . hal-00513777

\section{HAL Id: hal-00513777 \\ https://hal.science/hal-00513777}

Submitted on 1 Sep 2010

HAL is a multi-disciplinary open access archive for the deposit and dissemination of scientific research documents, whether they are published or not. The documents may come from teaching and research institutions in France or abroad, or from public or private research centers.
L'archive ouverte pluridisciplinaire HAL, est destinée au dépôt et à la diffusion de documents scientifiques de niveau recherche, publiés ou non, émanant des établissements d'enseignement et de recherche français ou étrangers, des laboratoires publics ou privés. 


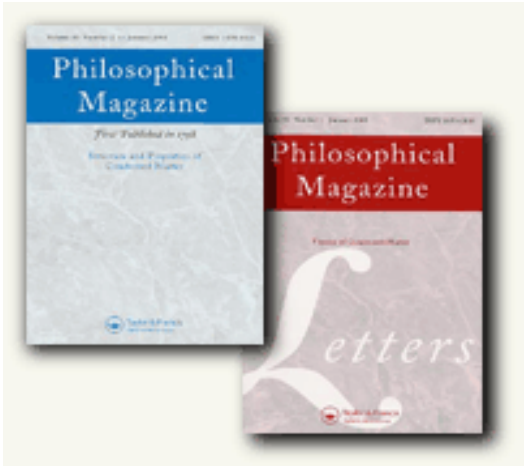

\section{Probing the cooperative dynamics varying the side-chain length of poly(alkyl acrylate)s: ESR experiments}

\begin{tabular}{|r|l|}
\hline Journal: & Philosophical Magazine \& Philosophical Magazine Letters \\
\hline Manuscript ID: & TPHM-06-Apr-0133.R1 \\
\hline Journal Selection: & Philosophical Magazine \\
\hline Author: & 12-Jul-2006 \\
\hline & $\begin{array}{l}\text { Complete List of Authors: } \\
\text { Autiero, Ciro; University of Pisa, Physics } \\
\text { Giordano, Marco; University of Pisa, Physics } \\
\text { Zulli, Fabio; University of Pisa, Physics }\end{array}$ \\
\hline Keywords: & EPR, polymers \\
\hline Keywords (user supplied): & cooperativity, crossover, probe dynamics \\
\hline & \multicolumn{2}{|l}{} \\
\hline
\end{tabular}

\section{s scholaroNE" \\ Manuscript Central}


Probing the cooperative dynamics varying the side-chain length of PAAs

\title{
Probing the cooperative dynamics varying the side-chain length of poly(alkyl acrylate)s: ESR experiments
}

\author{
Laura Andreozzi* , Ciro Autiero, Massimo Faetti, Marco Giordano, Fabio Zulli \\ Department of Physics 'E. Fermi', University of Pisa and polyLab-CNR, \\ largo Pontecorvo 3, 56127 Pisa, Italy
}

\begin{abstract}
The rotational dynamics of the tracer cholestane dissolved in unentangled nearly monodisperse poly(alkyl acrylate) melts has been investigated by means of electron spin resonance spectroscopy. Three samples of almost same molecular weight were selected, poly(methyl acrylate) poly(ethyl acrylate) and poly(n-butyl acrylate), whose linear viscoelastic properties were also characterised. Large temperature intervals were found with power laws relating shear flow relaxation and probe rotational diffusion. The main aim of this paper was the study of the sharp crossover between two different fractional regimes that for all the samples was observed at a temperature $T_{C}=1.15-1.25 T_{g}$. The decoupling from structural relaxation at $T<T_{C}$ was ascribed to the onset of cooperative effects on the length scale probed by the tracer. These results fit in the general scenario regarding the dynamical information extractable from ESR on cholestane, a probe with its long axis dimension greater than the typical length characterising the rigid segment of polymers.
\end{abstract}

Keywords: Cooperativity; Crossover; Polymers; Side-chain; Probe dynamics; ESR

\footnotetext{
*Author for correspondence. E-mail: laura.andreozzi@ df.unipi.it.
} 
Probing the cooperative dynamics varying the side-chain length of PAAs

\section{Introduction}

The comprehension of the mechanisms of relaxation in glass-forming materials in their supercooled state is an important still unresolved topic in condensed matter physics [1, 2]. The temperature scaling of relaxation times exhibits a pseudo-Arrhenius form $\tau_{\infty} \exp \left(E_{T} / k T\right)$ ( $k$ is the Boltzmann's constant) with a $T$-dependent activation energy $E_{T}$. The most widely employed Vogel-Fulcher (VF) [3] form shows an observation window in which, in many cases [4], a single set of VF parameters is not able to give account of the experimental relaxation time data, and its coefficients have to vary around a certain temperature. This suggests that the mechanism of slow structural relaxation makes some alterations there. In particular, dynamical changes take place in a narrow range of temperatures typically located at about 1.1-1.2 $T_{g}$ and have been often observed with different spectroscopies [5-7]. These phenomena have been often ascribed to the onset of cooperative molecular rearrangements, and to the effect of the dynamic heterogeneity developing on approaching the glass transition from above. When polymeric materials are considered, also polydispersity and topology could play important roles.

Several works suggested that the length scale characterising the heterogeneity and the cooperative regions is of the order of the nanometer $[5,8,9]$. This represents one of the reasons of the appeal exerted by molecular probe spectroscopy, as its recent application in the framework of dielectric spectroscopy also testifies [10]. With time resolved fluorescence measurements of doped malachite green molecules dissolved in different glass formers [11] and with positron annihilation lifetime spectroscopy studies [12] dynamic anomalies were enlightened in the range 1.15-1.4T .

As far as the study of this crossover region is concerned, electron spin resonance spectroscopy (ESR) deserves consideration, due to its well-known sensitivity on the nanometer length scale and nanosecond time scale. ESR investigation of rotational dynamics of different paramagnetic tracers dissolved in low molecular weight glass formers and polymers evidenced the existence, in the 
Probing the cooperative dynamics varying the side-chain length of PAAs

supercooled region, of a dynamic crossover at about $1.2 T_{g}$ [13-16] and of partial coupling between probe rotation and shear viscosity [14-17].

In this work, we present ESR results on the temperature dependence of the rotation of the cholestane spin-probe dissolved in narrow distribution poly(alkyl acrylate) (PAA) samples of very comparable molar weight: a poly(methyl acrylate) (PMA), a poly(ethyl acrylate) (PEA), and a poly(nbutyl acrylate) (PnBA). The main aim of this work was to enlighten the influence of the side chain length on the dynamic anomalies observed in the crossover region. A linear viscoelastic characterisation of the samples is also presented.

\section{Materials and experimental details}

The molecular structure of the repeating unit of the PAAs differs in the number $n$ of the $\mathrm{CH}_{2}$ groups along the alkylic side-chain (figure 1a). The PMA $(n=0)$ and the PnBA $(n=3)$ samples were sealed by Polymer Source Inc. (Montreal, Canada), which provided molar mass data from size exclusion chromatography (SEC) using polystyrene standards. The PEA sample $(n=1)$ was synthesized as described in [16]. SEC calibrated with monodisperse polystyrene standards allowed the determination of the $M_{n}$ and the $M_{w}$ average masses and the polydispersity index $M_{w} / M_{n}$ [18]. Measurements of the glass transition temperature $T_{g}$ were obtained by differential calorimetric scans (Perkin Elmer DSC7). The thermograms were recorded on heating at $10 \mathrm{~K} / \mathrm{min}$ after quenching at $40 \mathrm{~K} / \mathrm{min}$. Molar mass data and the $T_{g}$ temperatures of the samples are given in table 1 .

[Insert figure 1 about here]

[Insert table 1 about here]

Linear viscoelastic measurements on the PAA samples were carried out with a Haake RS150H rheometer by using a parallel plate system (diameter $20 \mathrm{~mm}$ ). The gap between the plates was slightly 
Probing the cooperative dynamics varying the side-chain length of PAAs

increased on increasing temperature $(900-1100 \mu \mathrm{m})$ to account for the thermal dilatation. Iso-thermal frequency sweeps from $10^{-2}$ to $24.4 \mathrm{~Hz}$, and creep and flow measurements were performed to gain information on the zero-shear viscosity and the dynamic moduli.

X-band ESR measurements were performed on the probe-polymer systems by means of a Bruker ER200D SRC spectrometer equipped with the Bruker BVT100 temperature control apparatus with a nominal accuracy of $\pm 0.1 \mathrm{~K}$.

The stiff nitroxide molecule 3[beta]-doxyl-5[alpha]-cholestane (figure 1b) was used as paramagnetic probe. The samples were obtained mixing two solutions containing a predetermined amount of polymer and spin-probe. The resulting solution was evaporated to complete dryness $\left(10^{-4}\right.$ cholestane/repeating-unit molar ratio) in a standard ESR tube. Spectra in the ESR slow motion regime, close to $T_{g}$, were carefully recorded, allowing one to measure spectral distances with sensitivity of $10^{-2}$ gauss (via a NMR gaussmeter ER035M). The principal components of the magnetic Zeeman (g) and hyperfine (a) tensors [19] of the spin-probe in PAA samples were drawn by the powder ESR spectrum recorded at $140 \mathrm{~K}[20]$. The components of $\mathbf{g}$ and $\mathbf{a}$ of cholestane, identical for the probe dissolved in the different PAAs, are reported in table 2 in the molecular frame. These values determine the response of the system through the spin Hamiltonian: $H=H_{s}+H_{s B}+H_{B} \cdot H_{s}$ is the Hamiltonian invariant under rotation, $H_{B}$ describes the rotational degrees of freedom of the thermal bath and $H_{s B}$ couples the spin and orbital variables. The analysis of the ESR spectra is well established [21-23]. Numerical simulations based on the generalised Mori theory allow one to extract dynamic information from the experimental line-shapes [24].

[Insert table 2 about here]

\section{Results}


Probing the cooperative dynamics varying the side-chain length of PAAs

Figure 2 shows the temperature dependence of the zero-shear viscosity $\eta$ of the PAA samples, obtained from creep, flow and dynamic measurements. The $\eta$ temperature dependence of each sample is well reproduced (figure 2) by a unique Vogel-Fulcher law [3] in the whole temperature range:

$$
\eta(T)=\eta_{\infty} \exp \left(\frac{T_{b}}{T-T_{0}}\right)
$$

The values of the prefactors $\eta_{\infty}$, the pseudo-activation energies (expressed in kelvin) $T_{b}$, and the Vogel temperatures $T_{0}$ are reported in table 3 .

[Insert figure 2 about here]

[Insert table 3 about here]

In the whole investigated range, from $T_{g}+10 \mathrm{~K}$ up to $T_{g}+100 \mathrm{~K}$, the time-temperature superposition (TTS) principle was found to be applicable. The horizontal and vertical shift factors $a_{T r}$ and $b_{T r}$ at the reference temperature $T_{r}(279 \mathrm{~K}, 270 \mathrm{~K}$, and $259 \mathrm{~K}$ respectively for PMA, PEA, and PnBA), as well as the master curves, are obtained in this work by mathematically shifting the experimental isotherm frequency sweeps of the complex modulus $G^{*}(\omega)$ (figure 3 ).

[Insert figure 3 about here]

The PAA samples behave in a thermo-rheological simple way and the shift factors $a_{T r}(T)$ are well described by the Williams-Landel-Ferry (WLF) law [25]:

$\log a_{T r}(T)=\frac{-c_{1}\left(T-T_{r}\right)}{T-T_{r}+c_{2}}$

The values of the $c_{1}$ and $c_{2} T_{r}$-depending parameters, as well as the temperature invariants $c_{1} c_{2} \ln 10$, $T_{r}-c_{2}$, and the $c_{1}^{g}$ and $c_{2}^{g}$ parameters extrapolated at $T_{g}$ are given in table 3 . All the samples, within the experimental errors, fulfil the relationships $T_{b}=c_{1} c_{2} \ln 10$ and $T_{0}=T_{r}-c_{2}$, relating VF and WLF parameters if TTS holds and in the framework of the free volume approach. 
Probing the cooperative dynamics varying the side-chain length of PAAs

No evidence of rubbery plateau was found (according to the minimum $\tan \delta$ criterion), as expected for polymer masses of the order of the entanglement mass $M_{e}$ [26]. In the lowest frequency region, the curves show the behaviour of monodisperse linear polymers $G^{\prime} \propto \omega^{2}$ and $G^{\prime \prime} \propto \omega$. The wide range of frequencies in which the moduli of PMA are quite parallel follows from the fact that the ratio between the molar and the entanglement mass for the PMA sample is higher than for the other samples.

The ESR measurements on PAAs were carried out in the temperature range from $205 \mathrm{~K}$ to 410 K. Because of its nearly axial symmetry, the cholestane probe exhibits an anisotropic rotational diffusion described in terms of spinning $\tau_{\|}$and tumbling $\tau_{\perp}$ correlation times. For the studied temperatures, the anisotropy ratio $\tau_{\|} / \tau_{\perp}$ varied from 12 to 20 on cooling.

In figure 4 the temperature dependence of $\tau_{\|}$is reported for all the PAA samples. Different dynamic regions can be identified, which are detailed in table 4. Figure 5 shows data for PnBA only. In each region, the correlation time is well represented assuming either the Arrhenius behaviour (regions I and IV)

$\tau_{\|}(T)=\tau_{\| \infty} \exp \left(\frac{\Delta E}{k T}\right)$

or the Vogel-Fulcher (regions II and III) one

$$
\tau_{\|}(T)=\tau_{\| \infty} \exp \left(\frac{T_{b}}{T-T_{0}}\right) .
$$

The fit parameters pertinent to the different regions of the PAA samples are given in table 4 .

[Insert figure 4 about here]

[Insert figure 5 about here]

[Insert table 4 about here]

\section{Discussion}


Probing the cooperative dynamics varying the side-chain length of PAAs

The discussion of region I can be found in [27], where the results of the PnBA and PEA samples were analysed with data of other four poly(ethyl acrylate)s samples. A dependence of the onset temperature $T_{A}$ with the mass for PAAs was inferred, able to account of the lack of crossover to region I in the investigated $T$-range for PMA.

An activated regime (region IV) is also found on lowering the temperature down to the glassy state. The values of the activation energies (table 4) suggest that the rotational dynamics is coupled to secondary relaxation processes of PAAs. In particular, for PEA and PMA, $\Delta E$ agrees with the activation energy of secondary $\beta$ relaxation found by dielectric spectroscopy and ascribed to the dynamic of the -COOR side chain [28], while for PnBA, $\Delta E$ coincides with the activation energy of the relaxation of the -OR group in the side-chain [29].

A different mechanism drives the probe rotational relaxation in the other two dynamical regions II and III. There, the rotational diffusion is well described by VF laws characterised by the same value of the Vogel temperature $T_{0}$. Such a value is also coincident, within the experimental errors, with the $T_{0}$ value found for the shear viscosity (table 3). Accordingly, the probe dynamics appears to be coupled with the collective dynamics of the polymer that is responsible for the glass transition. For all the investigated samples, the crossover between the two regimes takes place at a temperature $T_{C}$ located in the range $1.15-1.25 T_{g}$ (see table 5) that well agrees with the critical temperature range of the mode coupling theory [30], and with related recent experimental studies [7] in polymers. In this temperature interval, crossovers between different dynamic regimes in glass-formers are also a current finding of various spectroscopies $[5,6,11,12]$. The above result on PAAs intrigues and proposes the ESR as a major spectroscopy to investigate the crossover dynamics. Actually, similar dynamic anomalies, related to the occurrence of a larger decoupling from the structural relaxation of the host matrix, were found for the cholestane rotation in other polymers [14-16] whereas, with the smaller paramagnetic probe 
Probing the cooperative dynamics varying the side-chain length of PAAs

tempo, the $\alpha$ - $\beta$ splitting of the relaxation was observed in PVAc [13] and PPG4000 [31] at 1.2T . This also suggests that different dynamical features can be observed with probe spectroscopy, by selecting proper length scales [27].

[Include table 5 about here]

For all the PAA samples the value of the spinning correlation time at $T_{C}, \tau\left(T_{C}\right)$, is of the order of magnitude of the monomeric rotational correlation time $\tau_{m}$, as found from NMR measurements in polybutadiene and polyethylene-oxide solutions [32]. Interestingly enough, in this work $\tau_{m}$ resulted to be molecular weight independent for homologue polymer species, as found also elsewhere for $\tau\left(T_{C}\right)$ in PEAs [16]. Nevertheless, a weak dependence of $\tau\left(T_{C}\right)$ on the side-chain length can be here appreciated. The bigger the monomeric unit the slower the time, in fact $\tau\left(T_{C}\right)$ values $\approx 30 \mathrm{~ns}$ in PMA, $\approx 33 \mathrm{~ns}$ in PEA, and $\approx 37 \mathrm{~ns}$ in PnBA.

[Include figure 6 about here]

By inspection of figure 6, one can appreciate the sharpness of the dynamic crossover seen by ESR spectroscopy. It also appears that the relation between guest rotational dynamics and host viscous flow follows fractional laws:

$$
\tau_{\|} \propto[\eta(T)]^{\xi} \quad 0<\xi \leq 1,
$$

that also were found to work in other ESR studies by us [14-17], and also with other kinds of probe spectroscopy [11].

The values assumed by the exponent of equation 5 for the PAAs in the regions II and III, for sake of clarity referred to as $\xi$ and $\xi^{\prime}$ respectively, are reported in table 5. They can be expressed as the ratio of the pseudo-activation energy of regions II or III over the one obtained from viscosity, and represent the coupling degree between probe dynamics and structural relaxation of the host [15]. The decoupling $(\xi<1)$ found in the region II has to be ascribed to steric hindrance effects of the guest-host geometry 
Probing the cooperative dynamics varying the side-chain length of PAAs

and interaction [15]. This statement seems to be supported by the results in PEA polymers [16], where $\xi$ was found to be independent of the polymer repeating unit, and in the present case of PAAs where the relation between the $\xi$ value and the side-chain length is consistent with a linear decreasing proportionality (figure 7a).

Below $T_{C}$ (region III), the change in the local polymeric dynamics effective on the length scale of the tracer [15] is observed, which has to be related to the onset of cooperative processes in the polymer chains [33], which are expected to be roughly independent on the side-chain length. However for the $\xi^{\prime}$ exponents a linear dependence with the mass of the repeating units $M_{0}$ of PAAs can be observed (figure 7b) that can be accounted for on the following basis. The $\xi^{\prime}$ values can be viewed as the product of a cooperative decoupling factor $\xi_{C}$ with the $\xi$ values coming from steric hindrance. Therefore, the ratio $\xi_{C}=\xi^{\prime} / \xi$ should represent the true cooperativity degree on the length scale of the molecular probe $[15,16]$, and it is actually found to be a constant for the PAAs (figure 7c) according this interpretation.

[Include figure 7 about here]

\section{Conclusions}

The dynamics of a nanometric molecular tracer in nearly monodisperse PAA samples of almost the same molecular weight has been studied by ESR spectroscopy. Different dynamical regimes with sharp crossovers have been recognised. In particular, two regions described by the scaling law of equation 5 with different decreasing exponents have been found. Moreover, we have verified that a side-chain independent mechanism is responsible of the crossover from a region to the other, and interpreted this as the onset of cooperativity. Furthermore, the coupling of the probe dynamics to local modes of the host was observed below a temperature $T_{G}$, close to the calorimetric glass transition. These results 
Probing the cooperative dynamics varying the side-chain length of PAAs

confirm a general scenario already singled out for the cholestane probe dissolved in other polymeric melts.

\section{Acknowledgements}

This work was partially supported by Italian MIUR and INFM Pais project.

\section{References}

[1] K. Ngai, J. Non-Cryst. Sol. 275 7-51 (2000).

[2] P.G. Debenedetti, F.H.Stillinger, Nature 410 259-267 (2001)

[3] H. Vogel, Z. Phys. 22 641-646 (1921). G.S. Fulcher, J. Am. Chem. Soc. 8 339-345 (1925). G. Tamman, W. Hesse, Z. Anorg. Chem. 156 245-257 (1926).

[4] C. Hansen, F. Stickel, T. Berger, R. Richert, E.W. Fisher, J. Chem. Phys. 107 1086-1093 (1997); R. Richert, C.A. Angell, J. Chem. Phys. 108 9016-9026 (1998).

[5] E. Donth, The Glass Transition (Springer-Verlag, Berlin, 2001).

[6] M. Beiner, H. Huth, K. Schroeter, J. Non-Cryst. Sol. 279 126-135 (2001).

[7] A. Kisliuk, R.T.Mathers, A.P. Sokolov, J. Polym. Sci. Part B: Polym. Phys. 38 2785-2790 (2000).

[8] L. Berthier, D. Chandler, J.P. Garrahan, Europhys. Lett. 69 320-326 (2005).

[9] H. Sillescu, J. Non-Cryrst. Sol. 243 81-108 (1999).

[10] O. van der Berg, M. Wübbenhorst, S.J. Picken, W.F. Jager, J. Non-Cryst. Sol. 351 2694-2702 (2005); R. Richert, J. Non-Cryst. Sol. 351 2716-2722 (2005).

[11] J.Y. Ye, T. Hattori, H. Nakatsuka, Phys. Rev. B 56 5286-5296 (1997).

[12] J. Bartos, O. Sausa, P. Bandzuch, J. Zrubcova, J. Kristiak, J. Non-Cryst. Sol. 307-310 417-425 (2002).

[13] M. Faetti, M. Giordano, D. Leporini, L. Pardi, Macromolecules 32 1876-1882 (1999). 
Probing the cooperative dynamics varying the side-chain length of PAAs

[14] L. Andreozzi, M. Faetti, M. Giordano, D. Palazzuoli, W. Vittiglio, V. Castelvetro, C. De Vita, Macromol. Chem. Phys. 203 1445-1453 (2002).

[15] L. Andreozzi, M. Faetti, M. Giordano, D. Palazzuoli, G. Galli, Macromolecules 34 7325-7330 (2001).

[16] L. Andreozzi, M. Faetti, M. Giordano, F. Zulli, V. Castelvetro, Philos. Mag. 84 1555-1565 (2004).

[17] L. Andreozzi, M. Faetti, M. Giordano, J. Phys.: Condens. Matter 18 931-940 (2006).

[18] L.H. Sperling, Introduction to Physical Polymer Science, third edition (Wiley, New York, 1996).

[19] J.H. Freed, in Electron Spin Relaxation in Liquids, edited by L.T. Muus, and P.W. Atkins P. W. (Plenum Press, New York, 1972), p. 387.

[20] L. Andreozzi, M. Giordano, D. Leporini, Appl. Magn. Res. 4 279-295 (1993).

[21] L.T. Muus, P.W. Atkins (editors), Electron Spin Relaxation in Liquids (Plenum Press, New York, 1972).

[22] L.J. Berliner (editor), Spin Labelling: Theory and Applications, vol 1 (Academic Press, New York, 1976). L.J. Berliner (editor), Spin Labelling: Theory and Applications, vol 2 (Academic Press, New York, 1979).

[23] C.P. Slichter, Principles of Magnetic Resonance (Harper \& Row, New York, 1965).

[24] M. Giordano, P. Grigolini, D. Leporini, P. Marin, Adv. Chem. Phys. 62 321-388 (1985).

[25] M.L. Williams, R.F. Landel, J.D. Ferry, J. Am. Chem. Soc. 77 3701-3707 (1955).

[26] R.G. Larson, T. Sridhar, L.G. Leal, G.H. McKinley, A.E. Likhtman, T.C.B. McLeish, J. Rheol. 47 809-818 (2003).

[27] L. Andreozzi, C. Autiero, M. Faetti, M. Giordano, F. Zulli, J. Phys.: Condens. Matter 18 64816492 (2006).

[28] N.G. McCrum, B.E. Read, G. Williams, Anelastic and Dielectric Effects in Polymeric Solids (Dover Pubblications Inc., New York, 1991). 
Probing the cooperative dynamics varying the side-chain length of PAAs

[29] T. Hayakawa, K. Adachi, Polym. J. 32 845-848 (2000).

[30] W. Goetze, J. Phys.: Condens. Matter 11 A1-A46 (1999), and references therein.

[31] J. Bartos, L. Andreozzi, M. Faetti, O. Sausa, D. Rachko, J. Kristiak, J. Non-Cryst. Sol. in press (2006).

[32] A. Guillermo, J.P. Cohen Addad, J. Chem. Phys. 116 3141-3151 (2002).

[33] M. Guenza, Phys. Rev. Lett. 88025901 (2002). 
1

2

3

4

5

6

7

8

9

10

11

12

13

14

15

16

17

18

19

20

21

22

23

24

25

26

27

28

29

30

31

32

33

34

35

36

37

38

39

40

41

42

43

44

45

46

47

48

49

50

51

52

53

54

55

56

57

58

59

60

$$
\begin{aligned}
& -\left[\mathrm{CH}_{2}-\mathrm{CH}\right]- \\
& \text { (a) } \mathrm{O}=\mathrm{C}-\mathrm{O}-\left(\mathrm{CH}_{2}\right)_{\mathrm{n}}-\mathrm{CH}_{3}
\end{aligned}
$$

(b)

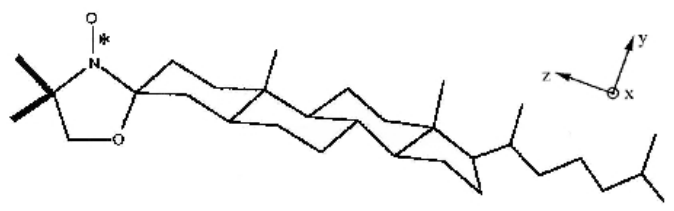

Figure 1. (a) Repeating unit of PAAs and (b) molecular structure of the cholestane spin-probe with its principal molecular frame. 


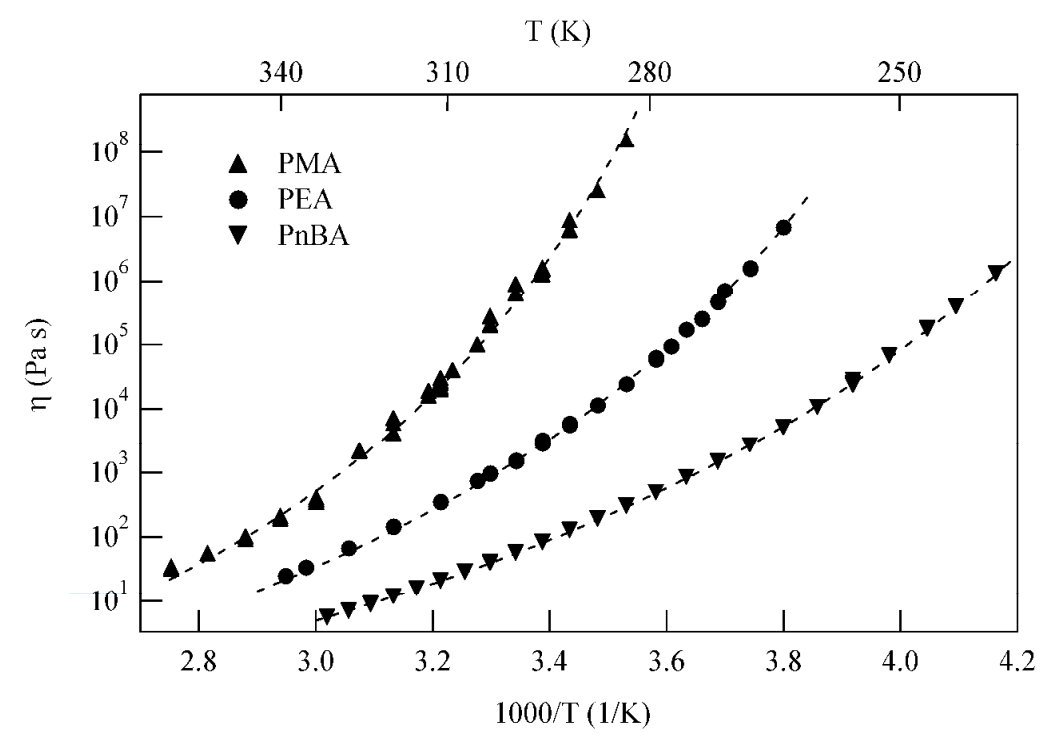

Figure 2. Temperature dependence of the zero-shear viscosity of PAA samples. 

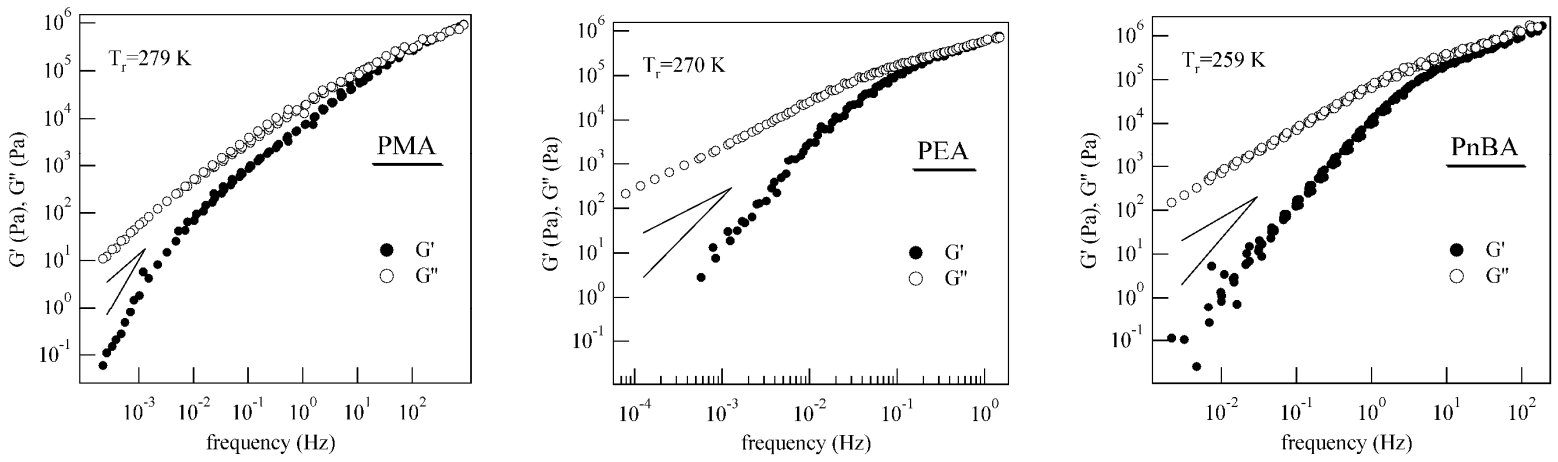

Figure 3. Master curves for the dynamic moduli of PAA samples at the reference temperature $T_{r}=279 \mathrm{~K}, 270 \mathrm{~K}$, and $259 \mathrm{~K}$ respectively for PMA, PEA, and PnBA. 


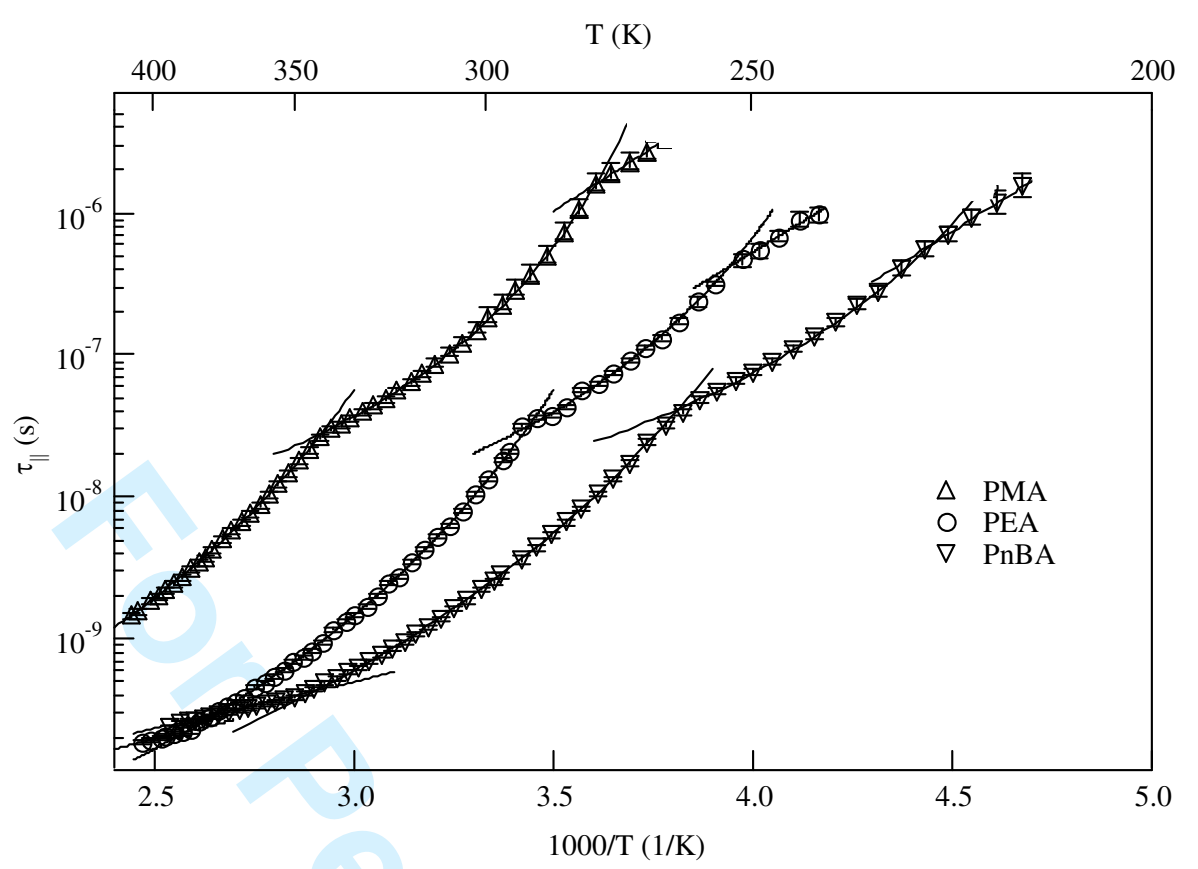

Figure 4. Temperature dependence of the spinning correlation time for cholestane in PAAs. 
1

3

5

6

7

8

9

10

11

12

13

14

15

16

17

18

19

20

21

IV) laws. 


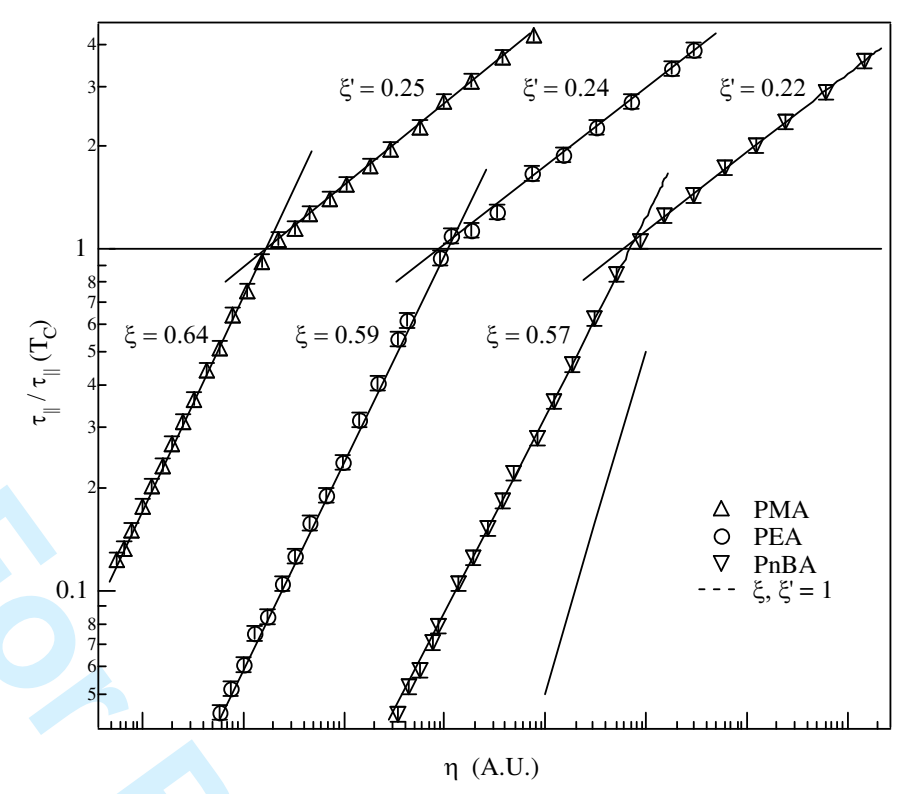

Figure 6. Power law dependence of the spinning correlation time as a function of viscosity for PAA samples. The curves are shifted horizontally to avoid overlap of the data. 


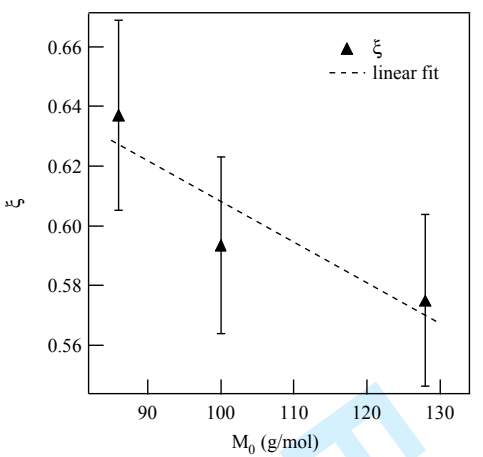

(a)

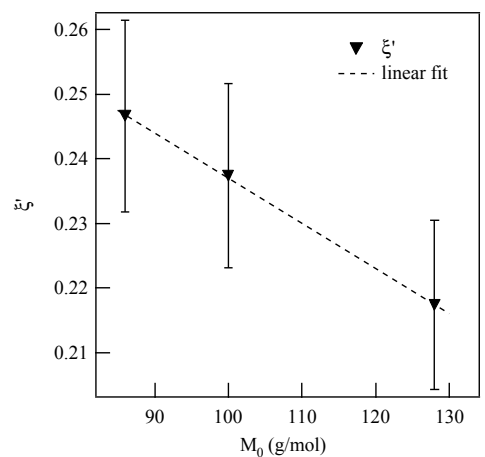

(b)

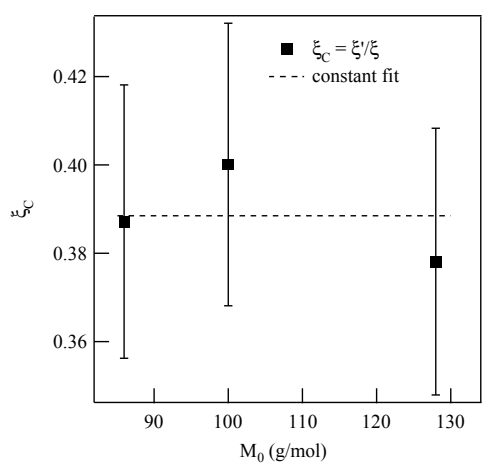

(c)

Figure 7. Dependence of the power law exponents (equation 5) and their ratio on the molar mass $M_{0}$ of the repeating unit of PAAs. 
Table 1. Molar mass data and glass transition temperatures $\left(T_{g}\right)$ of the PAAs. $M_{w} / M_{n}$ is the polydispersity index [18], $M_{0}$ the molar mass of the repeating unit, $M_{e}$ the entanglement mass, above which topological features in the dynamics set in [26].

\begin{tabular}{cccccccc}
\hline Sample & $n$ & $M_{n}(\mathrm{~g} / \mathrm{mol})$ & $M_{w}(\mathrm{~g} / \mathrm{mol})$ & $M_{w} / M_{n}$ & $M_{w} / M_{0}$ & $M_{e}(\mathrm{~g} / \mathrm{mol})$ & $T_{g}(\mathrm{~K})$ \\
\hline PMA & 0 & 13000 & 14000 & 1.08 & 163 & 11000 & 272 \\
PEA & 1 & 11600 & 13100 & 1.12 & 131 & 12000 & 252 \\
PnBA & 3 & 13000 & 14700 & 1.13 & 115 & $>13000$ & 224 \\
\hline
\end{tabular}


Table 2. Values of the components of the Zeeman and hyperfine tensors in the principal molecular reference frame for the cholestane probe in PAAs.

\begin{tabular}{cccccc}
\hline$g_{x x}$ & $g_{y y}$ & $g_{z z}$ & $a_{x x}(\mathrm{G})$ & $a_{y y}(\mathrm{G})$ & $a_{z z}(\mathrm{G})$ \\
\hline 2.0033 & 2.0092 & 2.0071 & 33.3 & 6.2 & 4.0
\end{tabular}


Table 3. VF and WLF parameters of PAA samples.

\begin{tabular}{ccccccccccc}
\hline Sample & $\eta_{\infty} \times 10^{-4}$ & $T_{b}$ & $T_{0}$ & $c_{1}$ & $c_{2}$ & $T_{r}$ & $c_{1} c_{2} \ln 10$ & $T_{r}-c_{2}$ & $c_{1}^{g}$ & $c_{2}^{g}$ \\
& $(\mathrm{~Pa} \mathrm{~s})$ & $(\mathrm{K})$ & $(\mathrm{K})$ & & $(\mathrm{K})$ & $(\mathrm{K})$ & $(\mathrm{K})$ & $(\mathrm{K})$ & & $(\mathrm{K})$ \\
& & & & & & & & & & \\
\hline PMA & $4.3 \pm 0.2$ & $1460 \pm 30$ & $229 \pm 4$ & 12.3 & 50 & 279 & $1410 \pm 50$ & $229 \pm 2$ & 14 & $43 \pm 4$ \\
PEA & $8.6 \pm 0.8$ & $1430 \pm 30$ & $200 \pm 4$ & 8.9 & 69 & 270 & $1410 \pm 50$ & $201 \pm 2$ & 12 & $52 \pm 4$ \\
PnBA & $6.6 \pm 0.2$ & $1425 \pm 30$ & $174 \pm 3$ & 7.2 & 86 & 259 & $1420 \pm 50$ & $173 \pm 2$ & 12 & $50 \pm 4$ \\
\hline
\end{tabular}


Table 4. Fit parameters for the temperature dependence of the spinning correlation time of cholestane in PAAs.

\begin{tabular}{|c|c|c|c|c|c|c|}
\hline \multirow[t]{2}{*}{ Sample } & \multirow[t]{2}{*}{ Zone } & Temperature Range & & \multicolumn{3}{|c|}{ Fit Parameters } \\
\hline & & $(\mathrm{K})$ & & $\begin{array}{c}\Delta E \\
(\mathrm{~kJ} / \mathrm{mol})\end{array}$ & $\begin{array}{c}T_{b} \\
(\mathrm{~K})\end{array}$ & $\begin{array}{l}T_{0} \\
(\mathrm{~K})\end{array}$ \\
\hline PMA & II & $340 \pm 2=T_{C}<T$ & VF & - & $930 \pm 40$ & $227 \pm 7$ \\
\hline PMA & III & $277 \pm 2=T_{G}<T<T_{C}=340 \pm 2$ & VF & - & $360 \pm 20$ & $227 \pm 7$ \\
\hline PMA & IV & $T<T_{G}=277 \pm 2$ & Arrh & $34 \pm 3$ & - & - \\
\hline PEA & I & $T>T_{A}=396 \pm 3$ & Arrh & $13 \pm 3$ & - & - \\
\hline PEA & II & $290 \pm 2=T_{C}<T<T_{A}=396 \pm 3$ & VF & - & $850 \pm 40$ & $201 \pm 6$ \\
\hline PEA & III & $253 \pm 2=T_{G}<T<T_{C}=290 \pm 2$ & VF & - & $340 \pm 20$ & $201 \pm 6$ \\
\hline PEA & IV & $T<T_{G}=253 \pm 2$ & Arrh & $35 \pm 3$ & - & - \\
\hline $\mathrm{PnBA}$ & I & $T>T_{A}=345 \pm 2$ & Arrh & $12.6 \pm 1.6$ & - & - \\
\hline $\mathrm{PnBA}$ & II & $262 \pm 2=T_{C}<T<T_{A}=345 \pm 2$ & VF & - & $820 \pm 40$ & $175 \pm 5$ \\
\hline $\mathrm{PnBA}$ & III & $225 \pm 2=T_{G}<T<T_{C}=262 \pm 2$ & VF & - & $310 \pm 20$ & $175 \pm 5$ \\
\hline $\mathrm{PnBA}$ & IV & $T<T_{G}=225 \pm 2$ & Arrh & $33 \pm 3$ & - & - \\
\hline
\end{tabular}


Table 5. Temperatures $T_{g}$ and $T_{C}$, ratios $T_{C} / T_{g}$, and $\xi$, $\xi$, and $\xi_{C}=\xi^{\prime} / \xi$ parameters.

\begin{tabular}{ccccccc}
\hline Sample & $T_{C} / T_{g}$ & $T_{C}(\mathrm{~K})$ & $T_{g}(\mathrm{~K})$ & $\xi$ & $\xi$ & $\xi_{C}=\xi^{\prime} / \xi$ \\
& & & & & & \\
\hline PMA & $1.25 \pm 0.02$ & 340 & 272 & $0.64 \pm 0.03$ & $0.25 \pm 0.02$ & $0.39 \pm 0.03$ \\
PEA & $1.15 \pm 0.02$ & 290 & 252 & $0.59 \pm 0.03$ & $0.24 \pm 0.02$ & $0.40 \pm 0.03$ \\
& & & & & & \\
PnBA & $1.17 \pm 0.02$ & 262 & 224 & $0.58 \pm 0.03$ & $0.22 \pm 0.02$ & $0.38 \pm 0.03$
\end{tabular}

